\title{
Infektionsbedingte Fruchtbarkeitsstörungen in schweizerischen Schweinezuchtbetrieben am Ende der «Postweaning Multisystemic Wasting Syndrome»(PMWS)-Epizootie
}

\author{
Handke, M ; Engels, M ; Prohaska, S ; Keller, C ; Brugnera, E ; Sydler, T ; Sidler, X
}

\begin{abstract}
Porzine Circoviren Typ 2 (PCV2) sind in der Lage Fruchtbarkeitsstörungen zu induzieren. Sechs Jahre nach Beginn der Epizootie des «Postweaning Multisystemic Wasting Syndrome» (PMWS) in der Schweiz wurden 286 Feten von 113 Muttersauen aus 59 Betrieben auf infektiöse Ursachen für Fruchtbarkeitsstörungen mit Aborten, vermehrt mumifizierten oder totgeborenen und lebensschwachen Ferkeln untersucht. $14 \%$ der Fälle wurden anhand von Erregerisolation und histologischen Entzündungsanzeichen einer bakteriellen Infektion zugeordnet. In weiteren $12 \%$ konnten histologisch Entzündungsreaktionen ohne plausiblen Erregernachweis gefunden werden. PCV2 wurde mittels Immunhistochemie (IHC) in nur $4 \%$ der Fälle nachgewiesen und scheint somit in der Schweiz bei Fruchtbarkeitsproblemen eine untergeordnete Rolle zu spielen. Infektionen mit dem porzinen Parvovirus (PPV) sind mit $3 \%$ der Fälle deutlich seltener als in früheren Untersuchungen. Auf Enteroviren/Teschovirus wurde nur stichprobenweise bei ätiologisch unklaren Fällen untersucht und eine Prävalenz von $11 \%$ ermittelt. Unseres Wissens ist das der erste Nachweis von Enteroviren/Teschovirus in Feten in der Schweiz. Die ätiologische Ursache blieb in über $50 \%$ aller Fälle, auch unter Anwendung moderner diagnostischer Methoden, unklar. = Porcine Circovirus type 2 (PCV2) is able to induce reproductive failures. 286 fetuses from 113 sows of 59 farms with increased reproductive disorders which included abortions, mummies, stillborn and weak born piglets were studied six years after the beginning of the epizooty of postweaning multisystemic wasting syndrome (PMWS) in Switzerland. $14 \%$ of the cases were bacterial infections based on histological signs of inflammation and pathogen isolation. $12 \%$ further cases showed inflammatory reactions by histology without pathogen identification. PCV2 was identified in only $4 \%$ of cases by immunohistochemistry (IHC). Thus, PCV2 infections are of minor importance in respect to pig reproductive failures in Switzerland. Porcine parvovirus (PPV) infections were found in $3 \%$ of the cases and seem to occur more infrequently compared to former findings. Hitherto, Enteroviruses/Teschovirus were marginally studied in etiologically undefined cases with a prevalence of $11 \%$. To our knowledge this is the first identification of Enteroviruses/Teschovirus in fetal tissue from reproductive failures in Switzerland. The etiology remained unclear in more than $50 \%$ of all cases in spite of modern diagnostic methods.
\end{abstract}

DOI: https://doi.org/10.1024/0036-7281/a000380

Other titles: Infection related fertility disorders in Swiss pig breeding farms at the end of the postweaning mulstisystemic wasting syndrome (PMWS) epizooty

Posted at the Zurich Open Repository and Archive, University of Zurich

ZORA URL: https://doi.org/10.5167/uzh-65898

Journal Article

Accepted Version 
Originally published at:

Handke, M; Engels, M; Prohaska, S; Keller, C; Brugnera, E; Sydler, T; Sidler, X (2012). Infektionsbedingte Fruchtbarkeitsstörungen in schweizerischen Schweinezuchtbetrieben am Ende der «Postweaning Multisystemic Wasting Syndrome» (PMWS)-Epizootie. Schweizer Archiv für Tierheilkunde, 154(10):437444.

DOI: https://doi.org/10.1024/0036-7281/a000380 
Infektiös

bedingte

Fruchtbarkeitsstörungen

in

schweizerischen

2 Schweinezuchtbetrieben am Ende der Postweaning Multisystemic Wasting Syndrom

3 (PMWS)-Epizootie

4

M. Handke ${ }^{1}$, M. Engels ${ }^{2}$, S. Prohaska ${ }^{3}$, Ch. Keller ${ }^{4}$, E. Brugnera ${ }^{1}$, T. Sydler ${ }^{5^{*}}$ und X. Sidler ${ }^{1 *}$

* gleichberechtigte Mitautoren

Zusammenfassung

Porzine Circoviren Typ 2 (PCV2) sind in der Lage Fruchtbarkeitsstörungen zu induzieren. Sechs Jahre nach Beginn der Epizootie des „Postweaning Multisystemic Wasting Syndroms“ (PMWS) in der Schweiz wurden 286 Feten von 113 Muttersauen aus 59 Betrieben auf infektiöse Ursachen für Fruchtbarkeitsstörungen mit Aborten, vermehrt mumifizierten oder totgeborenen und lebensschwachen Ferkeln untersucht. $14 \%$ der Fälle wurden anhand von Erregerisolation und histologischen Entzündungsanzeichen einer bakteriellen Infektion zugeordnet. In weiteren 12\% konnten histologisch Entzündungsreaktionen ohne plausiblen Erregernachweis gefunden werden.PCV2 wurde mittels Immunhistochemie (IHC) in nur 4\% der Fälle nachgewiesen und scheint somit in der Schweiz bei Fruchtbarkeitsproblemen eine untergeordnete Rolle zu spielen. Infektionen mit dem porzinen Parvovirus (PPV) sind mit 3\% der Fälle deutlich seltener als in früheren Untersuchungen. Auf Enteroviren/Teschovirus wurde nur stichprobenweise bei ätiologisch unklaren Fällen untersucht und eine Prävalenz von $11 \%$ ermittelt. Unseres Wissens ist das der erste Nachweis von Enteroviren/Teschovirus in Feten in der Schweiz. Die ätiologische Ursache blieb in über $50 \%$ aller Fälle, auch unter Anwendung moderner diagnostischer Methoden, unklar. 
Infectious related fertility disorders in Swiss pig breeding farms at the end of the postweaning mulstisystemic wasting syndrome (PMWS) epizooty

Summary

Porcine Circovirus type 2 (PCV2) is able to induce reproductive failures. 286 fetuses from 113 sows of 59 farms with increased reproductive disorders which included abortions, mummies, stillborn and weak born piglets were studied six years after the beginning of the epizooty of postweaning multisystemic wasting syndrome (PMWS) in Switzerland. $14 \%$ of the cases were bacterial infections based on histological signs of inflammation and pathogen isolation. $12 \%$ further cases showed inflammatory reactions by histology without pathogen identification. PCV2 was identified in only $4 \%$ of cases by immunohistochemistry (IHC). Thus, PCV2 infections are of minor importance in respect to pig reproductive failures in Switzerland. Porcine parvovirus (PPV) infections were found in 3\% of the cases and seem to occur more infrequently compared to former findings. Hitherto, Enteroviruses/Teschovirus were marginally studied in etiologically undefined cases with a prevalence of $11 \%$. To our knowledge this is the first identification of Enteroviruses/Teschovirus in fetal tissue from reproductive failures in Switzerland. The etiology remained unclear in more than $50 \%$ of all cases in spite of modern diagnostic methods.

Keywords: Reproductive failure, sow, prevalence, agents, PCV2,

Einleitung

Fruchtbarkeitsstörungen beim Schwein beinhalten verschiedene pathophysiologische Vorgänge und klinische Symptome. Sie können eingeteilt werden in (a) Störungen der Nidation (regelmässiges oder unregelmässiges Umrauschen), (b) frühzeitigen Trächtigkeitsabbruch mit Auswurf aller Feten (Abort) und

(c) reduzierte Anzahl lebend geborener Ferkel infolge nicht lebensfähiger Tiere wie Mumien, Totgeburten und lebensschwacher Ferkel (Almond et al., 2006). Die letzte Untersuchung zu schweizerischen 
Schweinezuchtbetrieben stammt aus den 90er Jahren (Broll et al., 1993). Damals konnte in 38\% aller untersuchten Fälle eine ätiologische Diagnose gestellt werden, wobei das porzine Parvovirus (PPV) mit 29\% der häufigste infektiöse Erreger war. Bakterien wurden in $8 \%$ aller Fälle nachgewiesen und fakultativ pathogene Erreger, wie E. coli und Streptokokken, standen an erster Stelle. In 10\% der Fälle gab es entzündliche Veränderungen an inneren Organen, jedoch konnte kein plausibler Erreger isoliert werden. Aus demselben Untersuchungsmaterial konnten in 4\% der Fälle zusätzlich Chlamydien in der Leber nachgewiesen werden (Thoma et al., 1997). In einer Datenzusammenstellung von 943 untersuchten Schweinefeten aus der Schweiz von 1988-1999 war PPV mit 15\% ebenfalls der am häufigsten nachgewiesene Erreger gefolgt von Bakterien (E. coli, Streptokokken und Mischinfektionen). In einem Fall wurden Leptospiren nachgewiesen (Pospischil et al., 2002). In 6\% der Fälle konnten entzündliche Veränderungen gefunden werden ohne dass ein Erreger isoliert werden konnte. In noch älteren Schweizer Untersuchungen wurde PPV mit 48\% (Zanoni et al., 1984) respektive 38\% (Brunner et al., 1987) als wichtigste infektiöse Ursache bei Fruchtbarkeitsstörungen gefunden. In den letzten Jahren hat das porzine Circovirus Typ 2 (PCV2) im Zusammenhang mit Fruchtbarkeitsstörungen beim Schwein weltweit zu Diskussionen Anlass gegeben. PCV2 Infektionen werden heute für eine ganze Reihe von Krankheiten verantwortlich oder mitverantwortlich gemacht, den sogenannten "Porcine Circovirus Associated Diseases" (PCVAD). Darunter fallen neben dem "Postweaning Multisystemic Wasting Syndrom" (PMWS), PCV2-assoziierte Pneumonien, Enteritiden, Lymphadenitiden, das "Porzine Dermatitis Nephropathie Syndrom" (PDNS) auch Fruchtbarkeitsstörungen (Opriessnig et al., 2007). Der erste Fallbericht von PCV2-assoziierten Fruchtbarkeitsstörungen stammt aus Kanada (West et al., 1999). Seither sind weltweit laufend neue Fallberichte dazugekommen, wobei PCV2 als alleiniger Infektionserreger gefunden (Josephson und Charbonneau, 2001; Meehan et al., 2001; Mikami et al., 2005; Brunborg et al., 2007; Pittman, 2008), oder als Koinfektion mit PPV, dem Encephalomyocarditis Virus (EMCV) und dem „Porcine Respiratory and Reproductive Syndrome Virus“ (PRRSV) (O'Connor et al., 2001; Pescador et al., 2007; Woods et al., 2009) in Mumien und abortierten Feten, sowie in

100 totgeborenen oder lebensschwachen Ferkeln nachgewiesen werden konnte. 101 PRRSV, das Pseudorabiesvirus (PRV) als Verursacher der Aujeszky'schen Krankheit 102 und die Europäische Schweinepest (ESP) gelten als wichtige Ursachen von 
103 Fruchtbarkeitsstörungen beim Schwein (Almond et al., 2006). Die Schweiz ist jedoch bezüglich dieser drei Krankheiten anerkannt frei (Schwermer und Sievi 2010). Sowohl porzine Enteroviren (PEV) als auch porzine Teschoviren (PTV) werden als Verursacher von Fruchtbarkeitsstörungen beim Schwein diskutiert (Knowles, 2006). Sie wurden im Zusammenhang mit dem "Stillbirth Mummification Embryonic Death and Infertility Syndrome" (SMEDI-Syndrom) (Dunne et al., 1965) und Abortfällen beim Schwein beschrieben (Bielanski und Raeside, 1977; Kirkbride und McAdaragh, 1978). Die Gruppe der porzinen Teschoviren umfasst die ehemaligen Serotypen 1-7 (Talfangruppe) der porzinen Enteroviren (Kaku et al., 2001). Von den bekannten

112 Serotypen gelten nur PTV 1, 3 und 6, sowie PEV 8 als pathogen für Feten (Knowles, 113 2006).

114 Die PMWS-Epizootie in der Schweiz begann Ende 2003 (Wiederkehr et al., 2009).

115 Der erste Fall von PCV2-bedingten Fruchtbarkeitsstörungen wurde in einem 116 Abferkelringbetrieb mit vermehrt Mumien und Totgeburten aber erst im Jahr 2008 117 diagnostiziert (Sydler et al., 2011). Ziel dieser Arbeit war es, sich ein aktuelles Bild 118 über die Ursachen von Fruchtbarkeitsstörungen in schweizerischen 119 Schweinezuchtbetrieben $\mathrm{zu}$ verschaffen. Sechs Jahre nach Beginn der 120 schweizerischen PMWS-Epizootie sollte insbesondere die Rolle von PCV2 im

121 Zusammenhang mit Fruchtbarkeitsstörungen genauer untersucht werden.

123 Material und Methoden

Herkunft des Probenmaterials

126 Von Januar bis Oktober 2009 wurden Feten und Plazenten aus 127 Schweinezuchtbetrieben mit Fruchtbarkeitsstörungen gesammelt. Das 128 Untersuchungsmaterial stammte aus Betrieben mit vermehrt auftretenden Aborten 129 (frühzeitiger Trächtigkeitsabbruch vor dem 110. Trächtigkeitstag mit gleichzeitigem 130 Auswurf aller Feten) oder einer erhöhten Anzahl an mumifizierten, autolytischen, 131 totgeborenen oder lebensschwachen Ferkeln. Pro Betrieb wurden maximal von drei 132 Muttersauen im gleichen Trächtigkeitsstadium Ferkel und Plazenten untersucht. Bei 133 Problemen in verschiedenen Trächtigkeitsstadien wurden pro Stadium zwei 134 Muttersauen mit Ferkeln untersucht. War es nicht möglich die Plazenta einem Feten 135 zuzuordnen, wurde von dieser an 3 verschiedenen Stellen Proben für die weiteren 136 Untersuchungen entnommen. Pro Muttersau wurden in der Regel 3 Feten 
137 untersucht, wobei, sofern vorhanden, Mumien und ein nicht-autolytischer Fetus in die 138 Untersuchungen einbezogen wurden. Für serologische Tests wurde den Muttertieren 139 mit Fruchtbarkeitsproblemen aus der Vena jugularis Blut entnommen.

141 Probenaufbereitung

142 Die Ferkel wurden gewogen und die Scheitel-Steiss-Länge (SSL) gemessen. Das

143 Gewebe wurde im Rahmen der Routinediagnostik sowohl pathologisch-anatomisch 144 als auch auf Bakterien und Viren untersucht. Für die Histologie wurden 145 Gewebeproben von Herz, Lunge, Leber, Niere, Milz, Mesenteriallymphknoten,

146 Thymus, Gehirn und Plazenta entnommen. Sie wurden in 4\%iger 147 Formaldehydlösung fixiert, in Paraffin eingebettet und Hämatoxilin-Eosin (HE) 148 gefärbt. in 10\%-igemFormalin fixiert, dann in Paraffin eingebettet und zu 2-3 $\mu \mathrm{m}$ 149 dicken Hämatoxilin-Eosin (HE) gefärbten Histologieschnitten weiter verarbeitet. 150 Folgende Spezialfärbungen wurden durchgeführt: eine modifizierte Gramfärbung 151 (Brown Brenn), Versilberung nach Warthing-Starry (W+S), Ziehl-Neelsen-Färbung 152 zur Darstellung säurefester Bakterien, von Kossa-Färbung zur Darstellung von 153 Kalksalzen und Van Gieson-Färbung zur Darstellung von Kollagenfasern. 154 Plazentaausstriche wurden mittels Köster- und Giménez-Färbung auf Infektionen mit 155 Brucellen und Chlamydien untersucht. Von den Plazenten und den inneren Organen 156 wurde der mesophile, aerobe Keimgehalt auf Columbia-Blutagar mit 5\% 157 defibriniertem Schafblut (Oxoid, Pratteln, Schweiz) bestimmt. Die Differenzierung der 158 Bakterienisolate erfolgte mittels kulturell-biochemischen und molekularbiologischen 159 Methoden (Quinn et al., 1994). Lebernekrosen mit Verdacht auf eine 160 Leptospireninfektion wurden mittels PCR (IVD GmbH Hannover) und bei Verdacht 161 auf eine Mykobakterieninfektion ebenfalls mittels PCR (Kim et al. 2001) weiter 162 untersucht. Der PPV-Nachweis wurde aus einem Gewebehomogenat, bestehend 163 aus Herz, Lunge, Leber, Niere mittels Immunelektronenmikroskopie (IEM) und der 164 PPV-Antikörper-Nachweis mittels indirekter Immunfluoreszenz (IIF) durchgeführt 165 (Zanoni et al., 1984).

166 Der PCV2-Nachweis geschah mittels Immunhistochemie (IHC) an Paraffinschnitten 167 mit Hilfe des monoklonalen Antikörpers F217 (McNeilly et al., 2001; Staebler et al., 168 2004). Für den Enteroviren-Nachweis wurde je ein Ferkel von 44 verschiedenen 169 Muttersauen (27 Aborte und 17 Würfe mit vermehrt mumifizierten Ferkeln) 170 ungeklärter Ursache von Fruchtbarkeitsstörungen ausgewählt. Das für die PPV- 
171 Diagnostik verwendete tiefgefrorene Gewebehomogenat wurde mittels PCR (Zell et 172 al., 2000) von der Firma bioScreen (bioScreen European Veterinary Disease

173 Management Center $\mathrm{GmbH}$, Münster, Deutschland) auf porzine Teschoviren und 174 porzine Enteroviren Typen 8, 9 und 10 untersucht. PCV2-Templates im EDTA-Blut 175 der Muttersauen wurden mittels einer SYBR Green Technik (Publikation in 176 Vorbereitung) quantitativ gemessen.

178 Serologische Untersuchungen

179 Die Muttersauenseren wurden serologisch auf Antikörper folgender Erreger 180 untersucht: PCV2 mittels SERELISA ${ }^{\circledR}$ (SYNBIOTICS EUROPE SAS 2, Lyon, 181 Frankreich); Aujeszky`sches Virus mittels Herdcheck ${ }^{\circledR}$ Anti-PRVgB (IDEXX 182 Laboratories, Inc., IDEXX Switzerland, Liebefeld-Bern); PRRSV mittels HerdCheck ${ }^{\circledR}$ 183 PRRS ELISA 2XR (IDEXX Laboratories Inc., Westbrook, ME, USA); Leptospiren 184 mittels Micro-Agglutinationstest (MAT) (Serovare L. grippotyphosa, L. australis, L. 185 pomona, L. tarassovi, L. canicola, L. icterohaemorrhagiae und L. bataviae) und 186 Brucellen mittels dem Rose-Bengal-Test (RBT).

\section{Ergebnisse}

190 Insgesamt wurden 286 Ferkel von 113 Muttersauen/Fällen (eine Muttersau 191 repräsentiert einen Fall) aus 59 Betrieben untersucht. Das Untersuchungsmaterial 192 stammte aus 12 verschiedenen Kantonen, wobei Gebiete mit einer hohen 193 Schweinedichte (Luzern, Thurgau, St. Gallen und die Region Emmental im Kanton 194 Bern) mit 44 Betrieben (74.4\%) den Hauptteil der untersuchten Fälle darstellten. Bei 195 den 113 Fällen handelte es sich um 42 Aborte sowie um 71 zeitgerechte Geburten 196 mit mumifizierten, autolytischen, totgeborenen oder lebensschwachen Ferkeln (Tab 197 1).

199 Alle 113 Sauen waren serologisch negativ für PRRSV, PRV, Brucella suis und 200 Leptospiren. Histologisch wurde an keinem der Ferkel eine Enzephalitis beobachtet. 201 Bei den PCV2 IHC negativen Ferkeln konnte in keinem Fall eine Myokarditis 202 diagnostiziert werden. Es lagen somit auch keine Hinweise einer Beteiligung von 203 EMCV vor. Alle Plazenten waren mikroskopisch mittels Spezialfärbung negativ für 204 Chlamydien und Brucellen. 
In 33\% konnte entweder eine infektiöse Ursache (Erregernachweis in 21\% der Fälle)

206 oder histologisch entzündliche Veränderungen ohne Erregernachweis (in 12\% der

207 Fälle) gefunden werden. In über 50\% der Fälle fanden sich keine Hinweise auf ein 208 infektiöses Geschehen und in 4\% der untersuchten Fälle konnten Missbildungen und 209 Dystokien gefunden werden (Tab 2).

211 Viral bedingte Fruchtbarkeitsstörungen

212 Virale Abortursachen, ohne Einbezug von PEV beziehungsweise PTV, konnten mit $2137 \%$ der Fälle selten festgestellt werden. Die 5 mittels IHC diagnostizierten PCV2 214 Fälle stammten von 4 Jungsauen (3x 1. Wurf, 1x 2. Wurf) und einer Altsau (4. Wurf) 215 aus 4 verschiedenen Betrieben. In 3 Betrieben waren ausschliesslich Jungsauen (1. 216 und 2. Wurf) und in einem Betrieb alle Altersklassen betroffen. Die Muttersauen 217 ferkelten alle zwischen dem 114. und 118. Trächtigkeitstag ab. In den betroffenen 218 Würfen war insbesondere eine erhöhte Anzahl an mumifizierten Ferkeln zu 219 verzeichnen (Tab 3). Die Muttertiere zeigten zum Zeitpunkt der Geburt keine 220 klinischen Symptome. Keiner der Betriebe setzte eine PCV2-Mutterschutzimpfung 221 ein. Alle 5 Sauen hatten hohe Antikörpertiter gegen PCV2 jedoch konnten nur in 3 222 von 5 Muttersauen geringe Mengen von PCV2-DNA mittels PCR nachgewiesen 223 werden (Tab 4). Anhand der Scheitel-Steiss-Längen (SSL) wurden die 224 Todeszeitpunkte der Feten geschätzt. Kein Fetus starb vor dem 70. Trächtigkeitstag $225 \mathrm{ab}$ (Tab 4). Die mumifizierten oder stark autolytischen fetalen Organe konnten 226 histologisch kaum mehr hinsichtlich entzündlicher Veränderungen beurteilt werden, 227 da das Gewebe hochgradig ausgeblasst war und die Zellkerne oft nicht mehr 228 erkennbar waren. So konnten nur in 2 der 14 untersuchten Herzen eine Entzündung 229 und in 3 Herzen eine multifokale Fibrosierung zweifelsfrei diagnostiziert werden. 230 Allerdings wiesen 12 Herzen gut sichtbare multifokale dystrophische Verkalkungen 231 auf (Tab 5). Die PCV2-Antigenmengen in den verschiedenen fetalen Organen der 14 232 IHC-positiven Ferkel sind in Abb. 1 zusammengestellt. Im Myokard waren immer 233 mittel bis hochgradige PCV2-Antigen Mengen vorhanden. Auch in anderen Organen, 234 insbesondere Mesenteriallymphknoten, Thymus, Leber und Milz konnte PCV2235 Antigen in sehr hohen Mengen nachgewiesen werden. Überdies traten mittel- und 236 geringgradige Antigenmengen in diversen Feten in nahezu allen untersuchten 237 Organen auf. Antigenbeladene Zellen waren oft intravaskulär auch in Plazenten und 238 Gehirnen zu finden, aber auch als Einzelzellen oder Zellgruppen verteilt im 
Interstitium der Gewebe. Bei den intravaskulären PCV2-positiven Zellen handelte es

240 sich wahrscheinlich um Monozyten/Makrophagen und bei den interstitiellen Zellen

241 morphologisch um Histiozyten und dendritische Zellen. Myokardzellen und 242 Hepatozyten waren meist stark infiziert und diffus mit PCV2-positiven Zellen 243 überflutet. In den 3 untersuchten Thymi war das Mark stärker betroffen als die Rinde.

PPV konnte in 4 Mumien aus 3 Würfen (1. 3. und 7. Wurf) von 2 Beständen nachgewiesen werden. Im ersten Betrieb wurden die Muttersauen nach gängigem Impfschema gegen PPV vakziniert. Der betroffene Wurf bestand aus 2 Mumien, 2 Totgeburten und 10 lebensfähigen Ferkeln. PPV konnte nur in der untersuchten Mumie (SSL 11cm) nachgewiesen werden, nicht jedoch in den zwei untersuchten Totgeburten, die serologisch negativ für PPV waren. Im zweiten Betrieb wurde keine PPV-Impfung eingesetzt. Die beiden betroffenen Würfe bestanden hauptsächlich aus verschieden grossen Mumien, aber auch aus lebensschwachen und normalen, 253 lebensfähigen Ferkeln.

254 RNA von PEV beziehungsweise PTV konnte in 5 von 44 untersuchten Fällen mit bis anhin ungeklärter Ätiologie isoliert werden. Aus Würfen mit Mumien konnte 1x PTV und 2x PEV Typ 9 oder 10 nachgewiesen werden. PEV Typ 9 oder 10 wurde zudem auch in zwei Spätaborten ohne Mumien diagnostiziert. Die Muttersauen waren in allen Fällen ohne klinische Symptome.

Bakteriell bedingte Fruchtbarkeitsstörungen

261 In 14\% der Fälle konnten bakterielle Erreger zusammen mit entzündlichen 262 Veränderungen in Plazenta und oder in den fetalen Organen nachgewiesen werden. 263 Die Häufigkeiten der jeweils nachgewiesenen Erreger ist aus Tab 2 ersichtlich.

264 Mykobakterien wurden aus 2 Totgeburten isoliert, die aus einem Wurf am 116. 265 Trächtigkeitstag mit insgesamt 4 Totgeburten stammten. Histologisch konnte in 266 beiden Ferkeln eine hochgradige multifokal-konfluierende, nekrotisierende Plazentitis 267 und eine mittelgradige, multifokale, kleinherdförmige, granulomatöse Hepatitis jeweils mit Nachweis von massenhaft säurefesten Stäbchen nachgewiesen werden. Mittels PCR und nachfolgendem Restriktionsverdau konnten in den Proben Mykobakterien,

270 die nicht zum M. tuberculosis Komplex gehören, nachgewiesen werden. Der 271 Leptospirenverdachtsfall stammt von einem primiparen Muttertier, das am 98. 272 Trächtigkeitstag ohne weitere klinische Symptomatik abortierte. In 2 von 3 
273 untersuchten Ferkeln war die Leber makroskopisch mit $0.5-1 \mathrm{~cm}$ grossen, weiss274 grauen Flecken übersät. Histologisch handelte es sich dabei um eine mittelgradige 275 multifokale eitrig-nekrotisierende Hepatitis. Leichtgradige entzündliche 276 Veränderungen fanden sich auch in Lunge und Plazenta. Mittels Versilberung (W+S) 277 waren histologisch Leptospiren-ähnliche Erreger am Rand der Lebernekrosen 278 auffindbar. Die durchgeführte PCR zum Nachweis von Leptospiren fiel jedoch negativ 279 aus. Der Leptospiren-Antikörpertiter des Muttertieres lag für L. grippotyphosa bei $280 \quad 1: 100$.

Diskussion

Prävalenzangaben über PCV2-bedingte Fruchtbarkeitsstörungen variieren weltweit beachtlich, doch schwanken auch Sensitivität und Spezifität der angewandten Methoden stark. In einer amerikanischen Arbeit konnte PCV2 mittels PCR in keiner (Bogdan et al., 2001), in Spanien mittels PCR und in situ Hybridisierung in 1\% (Segales et al., 2002; Maldonado et al., 2005) und in Brasilien mit PCR und IHC in $5.7 \%$ (Pescador et al., 2007) der untersuchten Proben gefunden werden. Im Gegensatz dazu ergaben PCR-Untersuchungen aus Österreich eine PCV2Prävalenz von 20.5\% (Dastig et al., 2003) und aus Deutschland 27.1\% (Ritzmann et al., 2005b). In der Mitte liegt eine mittels PCR ermittelte Häufigkeit von $13.1 \%$ aus Korea (Kim et al., 2004). In unserer Arbeit konnte mittels IHC eine Prävalenz von 4\% ermittelt werden. Die Mehrheit der IHC-positiven Ferkel waren Mumien, von denen die meisten dystrophische Myokardverkalkungen aufwiesen. Um die Diagnose PCV2-assoziierte Fruchtbarkeitsstörungen stellen zu können, müssen gemäss Segales et al., (2006) drei Kriterien erfüllt sein: (I) klinische Symptomatik mit vermehrt Aborten und/oder Mumien und Totgeburten,

(II) Myokardveränderungen (Fibrosen/Degeneration und/oder Entzündung) und (III)

(III) PCV2-Nachweis in den 300 Läsionen. Die in dieser Arbeit erfassten Fälle erfüllen alle 3 Kriterien, wobei eine 301 Myokarditis infolge des schlechten Zustandes der mumifizierten Gewebe oft kaum ansprechbar war. Hingegen konnten Myokarddegeneration in Form dystrophischer Verkalkungen bei PCV2-bedingtem Fruchttod häufig beobachtet werden und sind auch im mumifizierten oder autolytischen Gewebe noch gut erkennbar. Während andere Autoren mittels IHC, PCR oder Virusisolation PCV2 auch in abortierten Feten finden konnten (Josephson und Charbonneau 2001; Meehan et al., 2001), wurde 
PCV2 Antigen in unserem Untersuchungsmaterial mittels IHC nur in Mumien und

308 einer Totgeburt nachgewiesen. Die Klinik entsprach den PPV-bedingten

309 Fruchtbarkeitsstörungen mit Vorhandensein von Mumien, aber auch

310 lebensschwachen und sich normal entwickelnden Ferkeln. In unseren

311 Untersuchungen waren ebenfalls, wie in der Literatur beschrieben, vor allem

312 Erstlingssauen und Jungsauen von PCV2-bedingten Fruchtbarkeitsstörungen

313 betroffen. Gefährdet sind Betriebe mit einem hohen Jungsauenanteil wie zum

314 Beispiel bei neu aufgebauten Sauenherden oder ein Wechsel des

315 Jungsauenlieferanten (West et al., 1999; Ladekjaer-Mikkelsen et al., 2001; O'Connor

316 et al., 2001; Josephson und Charbonneau, 2001; Sanford, 2002; Kim et al.,

317 2004;Brunborg et al., 2007;Pittman, 2008). Die Tatsache, dass vor allem Jungsauen

318 betroffen sind, kann mit einer mangelnden Immunität der Jungsauen wegen

319 ungenügender Akklimatisation an den neuen Standort erklärt werden. Im

320 vorliegenden Untersuchungsmaterial konnten bei einer Stichprobe mittels PCR im

321 Thymus auch bei IHC negativen Ferkeln PCV2 nachgewiesen werden. Nicht jede

322 intrauterine PCV2 Infektion führt demnach zwingend zu Fruchtbarkeitsstörungen.

323 Auch andere Autoren fanden in präkolostralen Blutproben bei rund $20 \%$ der

324 normalen lebensfähigen Ferkel mittels PCR geringe Mengen an PCV2 (Baker et al.,

325 2011). In einer weiteren Untersuchung konnte in präkolostralen Blutproben von

326 gesunden Neonaten in 21.4\% IgG gegen PCV2 und in 39.9\% PCV2-DNA

327 nachweisen werden (Shen et al., 2010). Eine latente vertikale Infektion ist

328 möglicherweise viel häufiger als bis anhin vermutet und könnte mit eine Erklärung für

329 die weite Verbreitung von PCV2 sein. Die Häufigkeit und Bedeutung latenter

330 intrauteriner Infektionen mit PCV2 muss weiter abgeklärt werden.

331 PPV wurde mit 3\% aller Fälle viel seltener nachgewiesen als dies noch in früheren

332 Untersuchungen der Fall war. Der markante Rückgang im Vergleich zu früheren

333 Angaben von Zanoni et al. 1984 (48\%) und Broll et al., 1993 (29\%) kann mit der

334 nahezu flächendeckend eingesetzten PPV-Impfung erklärt werden.

335 Bis anhin wurde unseres Wissens in der Schweiz bei Fruchtbarkeitsstörungen noch

336 nie auf PEV beziehungsweise PTV untersucht. Die gefundenen 5 Fälle aus einer

337 Stichprobe von 44 ätiologisch nicht geklärten Fällen mit Fruchtbarkeitsstörungen mit

338 vermehrten Mumien ergeben eine Prävalenz von 11.3\%. Allerdings ist nicht klar, ob

339 die nachgewiesenen Serotypen auch an den Fruchtbarkeitsstörungen ursächlich

340 beteiligt sind. In einer amerikanischen Untersuchung (Kirkbride und McAdaragh1978) 
341 war PEV mit $11 \%$ die am häufigsten gefundene infektiöse Ursache für 342 Fruchtbarkeitsstörungen beim Schwein. In unserem Untersuchungsmaterial konnte in 3434 der 5 Fälle PEV 9 und 10 nachgewiesen werden. Diese Serotypen wurden bis jetzt 344 nur in Italien, Grossbritannien und Japan beschrieben (Zoletto, 1965; Knowles et al., 345 1979; Caracappa et al., 1985; Honda et al., 1990). Sie werden aber in der Literatur 346 nicht mit Fruchtbarkeitsstörungen, sondern mit Hautläsionen in Zusammenhang 347 gebracht (Knowles et al., 1979). Eine systematische Untersuchung von 348 Fruchtbarkeitsproblemen auf PEV/PTV drängt sich auf.

349 Bei den bakteriellen Infektionen hat sich die Situation im Vergleich zu früheren 350 Untersuchungen nicht verändert. Fakultativ pathogene Keime, wie $E$. coli und 351 Streptokokken, stehen im Vordergrund. Brucellen konnten nicht nachgewiesen 352 werden und Leptospiren spielen im Gegensatz zu ausländischen Untersuchungen 353 (Kirkbride und McAdaragh 1978; Dastig et al., 2003) in der Schweiz bei 354 Fruchtbarkeitsproblemen kaum eine Rolle. In dieser Untersuchung standen häufig 355 auch Plazenten zu Verfügung. Trotzdem konnten im mikrosokpischen Ausstrich 356 keine Chlamydien nachgewiesen werden. Weitergehende Untersuchungen des 357 Materials auf Chlamydien oder Toxoplasmen mit IHC, PCR oder ELISA bleiben 358 nachfolgenden Arbeiten vorbehalten. 
375 Tabelle 1: Anzahl untersuchter Ferkel $(n=113)$ unterteilt nach Aborten, zeitgerechten 376 Geburten und Zustand der Ferkel.

\begin{tabular}{|l|c|c|c|}
\cline { 2 - 4 } \multicolumn{1}{c|}{} & $\mathbf{4 2}$ Aborte & $\begin{array}{c}\text { 71 zeitgerechte } \\
\text { Geburten }\end{array}$ & 113 Fälle \\
\cline { 2 - 4 } & 91 & 73 & 164 \\
\hline tote, normal entwickelte Ferkel & 4 & 55 & 59 \\
\hline Mumien & 6 & 11 & 17 \\
\hline autolytische Ferkel & 1 & 32 & 33 \\
\hline lebensschwache Ferkel & 13 & & 286 \\
\hline Frühaborte (<35.Trächtigkeitstag) & 115 & 171 & 13 \\
\hline Total untersuchte Ferkel & & & \\
\hline
\end{tabular}


401

\begin{tabular}{|l|c|c|c|}
\hline \multirow{2}{*}{ Ätiologie } & Erreger & Anzahl Fälle & $\%$ \\
\hline \multirow{2}{*}{\begin{tabular}{l} 
Viral \\
\cline { 2 - 4 }
\end{tabular}} & PCV2 & 5 & $4 \%$ \\
\cline { 2 - 4 } & Enteroviren/Teschoviren & 3 & $3 \%$ \\
\hline $\begin{array}{l}\text { Bnbekteriell } \\
\text { Hinweise für Infektion }\end{array}$ & & 16 & $14 \%$ \\
\hline $\begin{array}{l}\text { Missbildungen, } \\
\text { Dystokie }\end{array}$ & 14 & $12 \%$ \\
\hline $\begin{array}{l}\text { Keine Hinweise auf } \\
\text { infektiöses Geschehen }\end{array}$ & & 4 & $4 \%$ \\
\hline
\end{tabular}

402

${ }^{a}$ Untersuchung einer Stichprobe auf Enteroviren/Teschoviren aus 44 Würfen

${ }^{\mathrm{b}}$ Erregerisolation inkl. histologische Entzündungsanzeichen wie Plazentitis und oder Pneumonie. 
423 Tabelle 3: Wurfzusammensetzung der 5 PCV2-positiven Fälle.

424

\begin{tabular}{|c|c|c|c|c|c|c|}
\hline Sau & $\begin{array}{c}\text { Wurf- } \\
\text { nummer }\end{array}$ & $\begin{array}{c}\text { Gesamtzahl } \\
\text { Ferkel }\end{array}$ & Mumien & $\begin{array}{c}\text { Tot- } \\
\text { geburten }\end{array}$ & $\begin{array}{c}\text { Lebens- } \\
\text { schwache } \\
\text { Ferkel }\end{array}$ & $\begin{array}{c}\text { Normale } \\
\text { Ferkel }\end{array}$ \\
\hline $\mathbf{1}$ & 1 & 15 & 13 & 0 & 2 & 0 \\
\hline $\mathbf{2}$ & 1 & 10 & 6 & 0 & 0 & 4 \\
\hline $\mathbf{3}$ & 1 & 7 & 3 & 4 & 0 & 0 \\
\hline $\mathbf{4}$ & 2 & 13 & 10 & 1 & 1 & 1 \\
\hline $\mathbf{5}$ & 4 & 14 & 3 & 1 & 8 & 2 \\
\hline
\end{tabular}

425

426

427

428

429

430

431

432

433

434

435

436

437

438

439

440

441

442

443

444

445

446

447 
448 Tabelle 4: PCV2-Serologie und quantitative PCR bei den Muttersauen im Vergleich 449 zur Scheitel-Steiss-Länge (SSL) zum Todeszeitpunkt der PCV2-positiven Ferkel.

\begin{tabular}{|c|c|c|c|c|}
\hline $\begin{array}{c}\text { Fall- } \\
\text { Nr. }\end{array}$ & $\begin{array}{c}\text { Anzahl } \\
\text { Viren/mI } \\
\text { EDTA-Blut }\end{array}$ & $\begin{array}{c}\text { PCV2-IgG } \\
\text { Titer } \\
\text { Muttersau }\end{array}$ & $\begin{array}{c}\text { SSL der PCV2 } \\
\text { positiven Feten (cm) }\end{array}$ & $\begin{array}{c}\text { Todeszeitpunkt der } \\
\text { Feten in Trächtig- } \\
\text { keitstagen }\end{array}$ \\
\hline $\mathbf{1}$ & 0 & $>20000$ & $(\mathrm{M} / \mathrm{M}) 20 / 21$ & $83 / 86$ \\
\hline $\mathbf{2}$ & 0 & $>20000$ & $(\mathrm{M} / \mathrm{M} / \mathrm{A}) 17 / 21 / 29$ & $74 / 86 / 111$ \\
\hline $\mathbf{3}$ & $1.5 \times 10^{5}$ & 7102 & $(\mathrm{M} / \mathrm{A} / \mathrm{A}) 19 / 30 / 30$ & $80 / 114 / 114$ \\
\hline $\mathbf{4}$ & $5 \times 10^{5}$ & 6232 & $(\mathrm{M} / \mathrm{M} / \mathrm{T}) 24 / 29 / 29$ & $95 / 111 / 111$ \\
\hline $\mathbf{5}$ & $10^{5}$ & 4308 & $(\mathrm{M} / \mathrm{M} / \mathrm{T}) 29 / 29 / 30$ & $111 / 111 / 114$ \\
\hline
\end{tabular}

453 Der Todeszeitpunkt wurde mit der Formel nach Almond et al. (2006)berechnet:

$454 \quad$ Alter $=21.07+3.11 \times$ SSL 
473 Tabelle 5: Herzveränderungen der 14 PCV2 positiven Fälle.

474

\begin{tabular}{|l|c|c|c|c|c|c|}
\hline & \multicolumn{3}{|c|}{ Mumien (n=9) } & \multicolumn{3}{c|}{ Nicht-Mumien (n=5) } \\
\hline & ja & nein & n.b. ${ }^{\text {a }}$ & ja & nein & n.b. $^{{ }^{a}}$ \\
\hline Myokardverkalkung & 8 & 1 & & 4 & 1 & \\
\hline Myokardfibrose & 2 & 7 & & 1 & 4 & \\
\hline Myokarditis & 1 & & 8 & 1 & 2 & 2 \\
\hline
\end{tabular}

475

476 aufgrund Mumifikation oder starker Autolyse histologisch nicht beurteilbar (n.b.).

477

478

479

480

481

482

483

484

485

486

487

488

489

490

491

492

493

494

495

496

497

498

499

500

501 
502

503

504

505

506

507

508

509

510

511

512

513

514

515

516

517

518

519

520

521

522

523

524

Abbildung 1:



(1)


525 Abbildung 1: Prozentsatz (\%) der fetalen Organe mit unterschiedlicher Antigenmenge 526 von 14 IHC-positiven Ferkeln.

527

528 